\title{
Sustentabilidade de povoamentos de Acacia mearnsii De Wild. em diferentes idades: uma revisão da ciclagem de nutrientes
}

\section{Dione Richer Momolli ${ }^{1}$ e Mauro Valdir Schumacher ${ }^{2}$}

${ }^{1}$ Universidade Federal de Santa Maria. Centro de Ciências Rurais. Programa de Pós-Gradução em Engenharia Florestal. Camobi. Santa Maria-RS, Brasil (CEP 97105-900).E-mail: dionemomolli@gmail.com.

${ }^{2}$ Universidade Federal de Santa Maria. Centro de Ciências Rurais. Departamento de Ciências Florestais. Campus da UFSM. Laboratório de Ecologia Florestal. Camobi. Santa Maria-RS, Brasil (CEP 97105-900).

Resumo. A compreensão da ciclagem de nutrientes é uma importante ferramenta que auxilia nas atividades silviculturais, como é o caso da fertilização. Uma maneira direta de se avaliar a ciclagem de nutrientes é através de simulações de produção de biomassa e nutrientes e por meio do cálculo da eficiência de utilização biológica (EUB). Outra forma de avaliar se dá por meio da serapilheira produzida, que permite quantificar os estoques de nutrientes que serão decompostos com o passar do tempo, servindo como fonte nutricional de liberação lenta à presente e às futuras rotações. Diante disso, a proposta do trabalho é reunir estudos voltados à ciclagem de nutrientes em povoamentos de Acacia mearnsii De Wild. em diferentes idades.

Palavras-chave: Sustentabilidade; Serapilheira; Eficiência de utilização biológica.

\begin{abstract}
Sustainability of Acacia mearnsii stands at different ages: $A$ review of nutrient cycling. The understanding of nutrient cycling is an important tool that assists silvicultural activities, such as fertilization. A direct way of evaluating nutrient cycling is through simulations of biomass and nutrient production and by calculating the biological utilization efficiency (BUE). Another way of evaluating is through the litterfall, which allows quantifying the nutrient stocks that will be decomposed over time, serving as a nutritional source of slow release to present and future rotations. Therefore, the proposal of the work is to gather studies aimed at the nutrient cycling in Acacia mearnsii stands at different ages.
\end{abstract}

Keywords: Sustainability; Litter; Biological utilization efficiency.
Recebido: $11 / 12 / 2018$

Aceito:

$21 / 04 / 2019$

Publicado: $30 / 04 / 2019$

Acesso aberto

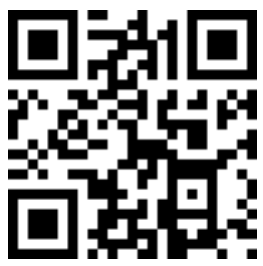

ORCID

(1) 0000-0001-7798-6355 Dione Richer Momolli

(D) 0000-0001-6926-6200

Mauro Valdir

Schumacher 


\section{Introdução}

Dentre as árvores plantadas, o gênero Acacia ocupa a quarta posição no Brasil, atrás apenas dos gêneros Eucalyptus, Pinus e Rubber. A área corresponde a 160 mil hectares (IBÁ, 2017) sendo desses, $56 \%$, ou 89,6 mil hectares situados no estado do Rio Grande do Sul (AGEFLOR, 2017).

A Acacia mearnsii De Wild. distribui-se naturalmente no sudeste da Austrália, com amplitudes de latitudes de $34^{\circ}$ a $43^{\circ}$ Sul (Boland et al., 1984). Nas últimas décadas tem se questionado a introdução da espécie, já que a mesma tem causado impactos aos ecossistemas em função de seu potencial de disseminação, que gera invasão e dominância sobre as espécies de ocorrência natural (Kull et al., 2011; Richardson e Rejmánek, 2011).

De acordo com Oliveira (1968), no Brasil, o primeiro plantio foi realizado no Estado do Rio Grande do Sul em 1918, sendo em 1930 iniciado em escala comercial. Atualmente são mais de $35 \mathrm{mil}$ famílias apenas no (RS), que dependem de forma direta ou indireta da acacicultura, sendo formada por pequenos e médios produtores (AGEFLOR, 2016).

Os plantios da espécie Acacia mearnsii, sob o ponto de vista comercial, fornecem madeira para geração de energia e com potencial uso para celulose e papel e da sua casca é obtido o tanino para curtimento do couro e peles (EMBRAPA, 2013). Com base no viés ambiental, os povoamentos promovem benefícios como a recuperação e proteção dos solos (Mochiutti et al., 2008), promovem condições biológicas ao desenvolvimento de micorrizas (Silva et al., 2008), e a fixação do nitrogênio por meio de rizóbios (Vargas et al., 2007).

Embora os povoamentos de acácia promovam a fixação de $\mathrm{N}_{2}$ atmosférico, o sistema de manejo é que definirá a sustentabilidade do sítio. Se compararmos com os povoamentos de Eucalyptus e Pinus, os mesmos visam apenas à obtenção da madeira como produto, deixando os chamados resíduos da colheita (casca + galhos + ponteira) distribuídos sobre o solo. Já a acácia, além da madeira, fornece a casca como importante subproduto, aumentando a exportação de nutrientes, o que implica em possíveis desequilíbrios no balanço nutricional ao longo das rotações.

Diante desses aspectos, o objetivo da presente revisão busca reunir dados de estoque de nutrientes nos compartimentos da biomassa, simulando exportações pela colheita florestal, além da deposição de nutrientes através da serapilheira.

Os plantios florestais concentram-se principalmente na metade sul do estado do Rio Grande Sul. Essas áreas são conhecidas por terem baixa fertilidade natural, e solos arenosos no bioma Pampa (Schumacher et al., 2013). Tratando-se de espécies arbóreas de rápido crescimento, o uso de fertilizantes minerais é essencial permitindo a planta expressar todo seu potencial genético, a fim de atender as demandas de produtividade e sustentabilidade desses sítios a rotações futuras. Caldeira et al. (2003) recomenda ainda que para haver manutenção da produtividade, a reposição nutricional deveria ser, pelo menos igual a exportada do sítio por meio da colheita.

Um dos meios de se obter respostas a elevadas produtividades se dá por meio da fertilização, nesse sentido, Andrade et al. (2004), determinaram as quantidades de fósforo e potássio que maximizam o crescimento da A. mearnsii em solos de baixa fertilidade no sudeste do Paraná. 0 povoamento estava situado em Latossolo Vermelho-Amarelo Distrófico típico, matéria orgânica de $3,5 \mathrm{mg} / \mathrm{dm}^{3}$ na camada de 0-20 cm. Os autores encontraram que a aplicação de 40 $\mathrm{g} /$ planta de $\mathrm{P}_{2} \mathrm{O}_{5}$ e $40 \mathrm{~g} /$ planta de $\mathrm{K}_{2} \mathrm{O}$ conjuntas, resultaram em melhores resultados de volume de madeira, representando ganhos entre 34\% e 70\% respectivamente. 
Outros estudos têm avaliado o crescimento da A. mearnsii em resposta a aplicação de N, P e K. Schumacher et al. (2013) avaliaram a resposta da acácia negra aos seis anos de idade em Argissolo Vermelho-Amarelo distrófico típico no município de Butiá-RS, a diferentes dosagens de $\mathrm{N}$ (ureia) $(0,20 \mathrm{e}$ $40 \mathrm{~kg} \mathrm{ha}), \mathrm{P}_{2} \mathrm{O}_{5}(0,50$ e $100 \mathrm{~kg} \mathrm{ha})$ e $\mathrm{K}_{2} \mathrm{O}$ $(0,25$ e $50 \mathrm{kgha})$ e concluíram que o máximo crescimento para a condição climática em questão se deu com a aplicação de $40 \mathrm{~kg}$ ha de nitrogênio e $80,0 \mathrm{~kg}$ ha de fósforo, sendo desnecessária a aplicação de potássio. Os autores ainda concluíram que o $\mathrm{P}$ foi $\mathrm{o}$ elemento mais importante para 0 crescimento das plantas.

Respostas tidas como lineares tem sido observada com a adição de fósforo sob as variáveis diâmetro a altura do peito (DAP), altura e volume. 0 potássio possui uma resposta menos expressiva sob as variáveis de crescimento. Tedesco (1999), com A. mearnsii não verificou efeito da adubação potássica para o crescimento inicial da espécie, já Mochiutti et al. (2008) mostraram que o potássio e o teor de matéria orgânica correlacionaram-se positivamente com a altura da A. mearnsii.

Estudando a influência da pedologia na produtividade de acácia-negra em Butiá-RS, Rachwal et al. (2008) concluíram que nos argissolos e cambissolos a produção de madeira foi $30 \%$ superior quando comparado com neossolo. Dessa forma, solos mais profundos, com boa drenagem e com maior disponibilidade de nutrientes, a acácia negra tem melhor desenvolvimento. Os autores ressaltam que pelo fato da acácia possuir sistema radicular superficial, a espécie torna-se susceptível ao tombamento e á danos no colo em função de ventos fortes quando a mesma for implantada em solos com muito cascalho.

\section{Produção e decomposição de serapilheira \\ Como estratégia ao baixo} suplemento de nutrientes pelo solo, as plantas, adaptam-se retranslocando seus nutrientes a outros tecidos (Inagaki et al., 2011). Em geral, a taxa de transferência de nutrientes entre os órgãos da planta tende a diminuir a medida em que o povoamento envelhece, sendo que quando ainda jovem, as taxas aumentam. Segundo Richards et al. (2010), isso pode ser ocasionado pelo desenvolvimento do sistema radicular e aumento da capacidade de obtenção de nutrientes do solo.

Avaliando a concentração e transferência de nutrientes das folhas em processo de senescência para as recém maduras em um povoamento de A. mearnsii aos três anos de idade, Caldeira et al. (1999) concluíram que em média, $40 \%$ dos macronutrientes são supridos pelo ciclo bioquímico. A magnitude de redistribuição dos nutrientes foi de $32 \%, 56 \%, 54 \%, 25 \%$ e $32 \%$ para N, P, K, Ca e Mg, respectivamente. Alguns estudos mostram que o $\mathrm{K}$ é um elemento facilmente lixiviado dos tecidos foliares (Pallardy, 2008; Torres e Pereira 2008), a precipitação pluviométrica pode ser um fator para redução da concentração do elemento nas folhas senescentes, que por sua vez acabam superestimando a redistribuição.

\section{Resultados e discussão}

Nas Tabelas 1 e 2, podemos observar a produção de serapilheira e retorno de nutrientes ao solo em plantios de acácia negra em diferentes idades. Podemos observar que do total de serapilheira produzida, $1 / 2$ são constituídos da fração foliar aos quatro anos e com o avançar da idade, 6 e 7 anos, a contribuição das folhas aumenta, passando a representar $3 / 4$. 
Tabela 1. Produção de serapilheira $\left(\mathrm{Mg} \mathrm{ha}^{-1}\right)$ nos diferentes componentes e idades.

\begin{tabular}{ccccccc}
\hline $\begin{array}{c}\text { Idade } \\
\text { (anos) }\end{array}$ & Folha & Galho & $\begin{array}{c}\text { Estrutura } \\
\text { reprodutiva } \\
\text { (Mg ha-1 }^{-1}\end{array}$ & Miscelânea & Total & Autor \\
\hline 4 & $4,1(48)$ & $1,9(22)$ & $1,79(21)$ & $0,7(9)$ & 8,5 & 1 \\
\hline 6 & $3,5(77)$ & - & $0,7(16)$ & $0,3(7)$ & 4,5 & 2 \\
\hline 7 & $3,1(75)$ & - & $0,3(7)$ & $0,8(18)$ & 4,1 & 1 \\
\hline 10 & - & - & - & - & 3,3 & 3 \\
\hline
\end{tabular}

1 - Silva et al. (2015); 2 - Viera et al. (2010); 3 - Forrester et al. (2005).

Tabela 2. Retorno de nutrientes $\left(\mathrm{kg} \mathrm{ha}^{-1}\right)$ ao solo via deposição de serapilheira em diferentes idades.

\begin{tabular}{|c|c|c|c|c|c|c|}
\hline \multirow{2}{*}{$\begin{array}{l}\text { Idade } \\
\text { (anos) }\end{array}$} & $\mathbf{N}$ & $\mathbf{P}$ & $\mathbf{K}$ & $\mathrm{Ca}$ & Mg & \multirow{2}{*}{ Autor } \\
\hline & \multicolumn{5}{|c|}{$\left(\mathrm{kg} \mathrm{ha}^{-1}\right)$} & \\
\hline 4 & 68,5 & 4,5 & 18,3 & 30,8 & 8,8 & 1 \\
\hline 6 & 71,2 & 2,7 & 24,0 & 22,5 & 8,3 & \\
\hline 7 & 78,5 & 2,1 & 12,9 & 23,7 & 7,6 & 2 \\
\hline 10 & 48,8 & 0,7 & - & - & - & 3 \\
\hline
\end{tabular}

1 - Silva et al. (2015); 2 - Viera et al. (2010); 3 - Forrester et al. (2005).

\section{Produção de biomassa e exportação de nutrientes}

Estudos voltados a estimativas da produção de biomassa em florestas nativas e plantações comerciais têm como finalidade a avaliação do incremento das florestas, quantificação de produtos e subprodutos de interesse comercial entre eles madeira, celulose, tanino e fins energéticos, além de melhorar a compreensão da ciclagem de nutrientes e no tocante sequestro de $\mathrm{CO}_{2}$ da atmosfera.

No que se refere a ciclagem e a exportação de nutrientes pela colheita, estudos prévios de biomassa e nutrientes, nos permitem inferir quanto a reposição via fertilização às futuras rotações. Esse manejo de reposição nutricional garante além da fertilidade do solo, a manutenção de um ecossistema balanceado e produtivo para as futuras rotações.
Na Tabela 3 podemos observar a produção de biomassa em (Mg ha-1) e em (\%) nos diferentes componentes de $A$. mearnsii sob diferentes idades e espaçamentos. A maturidade do povoamento é decisiva não apenas a quantidade de biomassa total, mas também aos percentuais de cada componente. A medida em que o povoamento envelhece, ocorre incremento em diâmetro e altura, resultando em produção de madeira e casca e diminuição da copa (principalmente de folhas e galhos vivos). Esse comportamento pode ser observado em estudo conduzido por Sanquetta et al. (2014), avaliando a produção de biomassa acima do solo de árvores de $A$. mearnsii nas idades $1,3,5$ e 7 anos com adubação de $50 \mathrm{~g}$ de $\mathrm{N}, \mathrm{P}_{2} \mathrm{O}_{5} \mathrm{e}$ $\mathrm{K}_{2} \mathrm{O}$ (5-30-15) por planta, em Cristal e Piratini-RS. 
Tabela 3. Biomassa nos diferentes componentes de Acacia mearnsii sob diferentes idades e espaçamentos.

\begin{tabular}{|c|c|c|c|c|c|c|c|c|c|c|}
\hline \multirow{2}{*}{$\begin{array}{l}\text { Idade } \\
\text { (anos) }\end{array}$} & \multirow{2}{*}{$\begin{array}{c}\text { Árvores } \\
\text { (ha-1) }\end{array}$} & Madeira & Casca & Folha & $\begin{array}{c}\text { Galho } \\
\text { vivo }\end{array}$ & $\begin{array}{l}\text { Galho } \\
\text { morto }\end{array}$ & $\begin{array}{l}\text { Flor e } \\
\text { Fruto } \\
\end{array}$ & Raiz & Total & \multirow[t]{2}{*}{ Autor } \\
\hline & & \multicolumn{8}{|c|}{$\left(\mathrm{Mg} \mathrm{ha}^{-1}\right)$} & \\
\hline 1,0 & 1904 & $\begin{array}{c}0,0 \\
(0,0)\end{array}$ & $\begin{array}{c}0,0 \\
(0,0)\end{array}$ & $\begin{array}{c}0,7 \\
(20,6)\end{array}$ & $\begin{array}{c}2,7 \\
(79,3)\end{array}$ & $\begin{array}{c}0,0 \\
(0,0)\end{array}$ & $\begin{array}{c}0,0 \\
(0,0)\end{array}$ & - & $\begin{array}{c}3,4 \\
(100,0)\end{array}$ & \multirow{4}{*}{1} \\
\hline 3,0 & 2222 & $\begin{array}{c}28,4 \\
(53,7)\end{array}$ & $\begin{array}{c}4,8 \\
(9,1)\end{array}$ & $\begin{array}{c}5,6 \\
(10,7)\end{array}$ & $\begin{array}{c}11,4 \\
(21,6)\end{array}$ & $\begin{array}{c}0,8 \\
(1,6)\end{array}$ & $\begin{array}{c}1,8 \\
(3,4)\end{array}$ & - & $\begin{array}{c}52,8 \\
(100,0)\end{array}$ & \\
\hline 5,0 & 2222 & $\begin{array}{c}66,3 \\
(66,4)\end{array}$ & $\begin{array}{c}9,8 \\
(9,8)\end{array}$ & $\begin{array}{c}4,5 \\
(4,6)\end{array}$ & $\begin{array}{c}13,0 \\
(13,1)\end{array}$ & $\begin{array}{c}4,9 \\
(4,9)\end{array}$ & $\begin{array}{c}1,2 \\
(1,2)\end{array}$ & - & $\begin{array}{c}99,7 \\
(100,0)\end{array}$ & \\
\hline 7,0 & 2222 & $\begin{array}{c}93,6 \\
(69,0)\end{array}$ & $\begin{array}{c}14,8 \\
(10,9)\end{array}$ & $\begin{array}{c}4,1 \\
(3,0)\end{array}$ & $\begin{array}{c}17,7 \\
(13,0)\end{array}$ & $\begin{array}{c}4,7 \\
(3,4)\end{array}$ & $\begin{array}{c}0,8 \\
(0,6)\end{array}$ & - & $\begin{array}{c}135,6 \\
(100,0)\end{array}$ & \\
\hline 4,0 & 2564 & $\begin{array}{c}46,6 \\
(63,9)\end{array}$ & $\begin{array}{c}7,1 \\
(9,7) \\
\end{array}$ & $\begin{array}{c}2,4 \\
(3,3)\end{array}$ & $\begin{array}{c}5,3 \\
(7,3) \\
\end{array}$ & $\begin{array}{c}3,4 \\
(4,6)\end{array}$ & - & $\begin{array}{c}8,1 \\
(11,1)\end{array}$ & $\begin{array}{c}72,9 \\
(100,0)\end{array}$ & 2 \\
\hline 8,0 & 1961 & $\begin{array}{c}82,4 \\
(62,3)\end{array}$ & $\begin{array}{l}10,9 \\
(8,3)\end{array}$ & $\begin{array}{c}3,9 \\
(3,0)\end{array}$ & $\begin{array}{l}12,4 \\
(9,4)\end{array}$ & $\begin{array}{c}6,1 \\
(4,6)\end{array}$ & - & $\begin{array}{c}16,5 \\
(12,5)\end{array}$ & $\begin{array}{c}132,1 \\
(100,0)\end{array}$ & 3 \\
\hline 1,0 & 5000 & $\begin{array}{c}2,1 \\
(40,1)\end{array}$ & $\begin{array}{c}0,5 \\
(10,5)\end{array}$ & $\begin{array}{c}1,2 \\
(22,3)\end{array}$ & \multicolumn{2}{|c|}{$\begin{array}{c}1,4 \\
(26,2)\end{array}$} & - & - & $\begin{array}{c}5,2 \\
(100,0)\end{array}$ & \multirow{4}{*}{5} \\
\hline 1,0 & $3333^{*}$ & $\begin{array}{c}1,1 \\
(34,8)\end{array}$ & $\begin{array}{c}0,2 \\
(7,4)\end{array}$ & $\begin{array}{c}0,9 \\
(29,3)\end{array}$ & \multicolumn{2}{|c|}{$\begin{array}{c}0,9 \\
(28,4)\end{array}$} & - & - & $\begin{array}{c}3,1 \\
(100,0)\end{array}$ & \\
\hline 1,0 & $3333^{* *}$ & $\begin{array}{c}0,8 \\
(30,0)\end{array}$ & $\begin{array}{c}0,2 \\
(7,1)\end{array}$ & $\begin{array}{c}1,0 \\
(38,6)\end{array}$ & \multicolumn{2}{|c|}{$\begin{array}{c}0,7 \\
(24,3)\end{array}$} & - & - & $\begin{array}{c}2,7 \\
(100,0)\end{array}$ & \\
\hline 1,0 & 2222 & $\begin{array}{c}0,6 \\
(33,0)\end{array}$ & $\begin{array}{c}0,1 \\
(4,1)\end{array}$ & $\begin{array}{c}0,8 \\
(39,7)\end{array}$ & \multicolumn{2}{|c|}{$\begin{array}{c}0,5 \\
(23,2)\end{array}$} & - & - & $\begin{array}{c}1,9 \\
(100,0)\end{array}$ & \\
\hline 3,0 & 5000 & $\begin{array}{c}45,0 \\
(45,9)\end{array}$ & $\begin{array}{c}14,2 \\
(14,5)\end{array}$ & $\begin{array}{c}10,8 \\
(11,0)\end{array}$ & \multicolumn{2}{|c|}{$\begin{array}{c}28,1 \\
(28,7)\end{array}$} & - & - & $\begin{array}{c}98,1 \\
(100,0)\end{array}$ & \multirow{4}{*}{5} \\
\hline 3,0 & $3333^{*}$ & $\begin{array}{c}24,6 \\
(39,1)\end{array}$ & $\begin{array}{c}7,0 \\
(11,2)\end{array}$ & $\begin{array}{c}9,5 \\
(15,2)\end{array}$ & \multicolumn{2}{|c|}{$\begin{array}{c}21,7 \\
(34,6)\end{array}$} & - & - & $\begin{array}{c}62,9 \\
(100,0)\end{array}$ & \\
\hline 3,0 & $3333^{* *}$ & $\begin{array}{c}17,7 \\
(35,7)\end{array}$ & $\begin{array}{c}3,3 \\
(6,6)\end{array}$ & $\begin{array}{c}9,3 \\
(18,6)\end{array}$ & \multicolumn{2}{|c|}{$\begin{array}{c}19,4 \\
(39,1)\end{array}$} & - & - & $\begin{array}{c}49,7 \\
(100,0)\end{array}$ & \\
\hline 3,0 & 2222 & $\begin{array}{c}7,3 \\
(27,6) \\
\end{array}$ & $\begin{array}{c}2,2 \\
(8,3) \\
-\end{array}$ & $\begin{array}{c}3,4 \\
(13,0)\end{array}$ & \multicolumn{2}{|c|}{$\begin{array}{c}13,5 \\
(51,1)\end{array}$} & - & - & $\begin{array}{c}26,4 \\
(100,0)\end{array}$ & \\
\hline 0,5 & 1666 & $\begin{array}{c}0,6 \\
(38,4)\end{array}$ & $\begin{array}{c}0,2 \\
(9,4)\end{array}$ & $\begin{array}{c}0,6 \\
(37,8)\end{array}$ & \multicolumn{2}{|c|}{$\begin{array}{c}0,2 \\
(14,5)\end{array}$} & - & - & $\begin{array}{c}1,6 \\
(100,0)\end{array}$ & 6 \\
\hline 0,9 & 1666 & \multicolumn{2}{|c|}{$\begin{array}{c}1,6 \\
(39,4)\end{array}$} & $\begin{array}{c}1,5 \\
(37,2)\end{array}$ & \multicolumn{2}{|c|}{$\begin{array}{c}1,0 \\
(23,4)\end{array}$} & - & - & $\begin{array}{c}4,1 \\
(100,0)\end{array}$ & 7 \\
\hline
\end{tabular}

Observações: * espaçamento $(2,0 \mathrm{~m}$ x 1,5 m); ** espaçamento (3,0 m x 1,0 m).

Valores entre " () ", referem-se aos percentuais de biomassa de cada componente.

1 - Sanquetta et al. (2014); 2 - Caldeira et al. (2011); 3 - Barichello et al. (2005); 5 - Caron et al. (2015); 6 - Viera et al. (2013); 7 - Kleinpaul et al. (2010).

Outro aspecto a ser levado em consideração é a densidade de plantas bem como a conformação do espaçamento ao qual o povoamento é estabelecido. Caron et al. (2015), estudando a $A$. mearnsii evidencia que a produção de biomassa total e madeira é diferente para mesma densidade populacional. Tanto no primeiro, quanto no terceiro ano de avaliação, a disposição das plantas resultou em uma maior produção de madeira quando submetidas ao tratamento $(2,0 \mathrm{~m} \times 1,5 \mathrm{~m})$ se comparado o tratamento $(3,0 \mathrm{~m} \times 1,0 \mathrm{~m})$, 
diferindo inclusive as médias a 5\% de probabilidade.

Segundo Schumacher et al. (2011), nos anos iniciais, a densidade populacional infere positivamente na biomassa por unidade de área, enquanto que a biomassa por indivíduo diminui, tendendo a estagnação conforme a maturação do povoamento.

Caldeira et al. (2014), mostram que a permanência dos resíduos da colheita (folhas, galhos, tocos entre outros), minimiza o exporte de nutrientes, já que os mesmos embora representem uma biomassa inferior, os teores de nutrientes desses componentes são elevados. Na Tabela 4 verifica-se a exportação de nutrientes por meio da colheita de madeira e casca de Acacia mearnsii sob densidades e idades diferentes.

A medida que em as árvores envelhecem, a exportação dos nutrientes aumenta por meio da retirada da madeira + casca. Caldeira et al. (2014), em estudo de biomassa em povoamentos de $A$. mearnsii com 0,5, 2,4, 4, 6 e 8 anos de idade mostra que a exportação de macronutrientes foi crescente a medida em que as árvores envelheciam, sendo de 13, 250, 481, 508 e $819 \mathrm{~kg} \mathrm{ha}^{-1}$, respectivamente.

Estudo desenvolvido por Caldeira et al. (2014), mostra que a manutenção dos resíduos (folha, raiz, galho morto e vivo) em povoamento de acácia negra com 4 anos, resulta na permanência de $57 \%, 56 \%, 49 \%, 29 \%, 41 \%$ e $45 \%$ de $\mathrm{N}$, $\mathrm{P}, \mathrm{K}, \mathrm{Ca}, \mathrm{Mg}$ e S, respectivamente.

Tabela 4. Exporte de macronutrientes $\left(\mathrm{kg} \mathrm{ha}^{-1}\right)$ na madeira e na casca de Acacia mearnsii sob diferentes idades.

\begin{tabular}{|c|c|c|c|c|c|c|c|}
\hline \multirow{2}{*}{$\begin{array}{l}\text { Idade } \\
\text { (anos) }\end{array}$} & \multirow{2}{*}{ Fração } & $\mathbf{N}$ & $\mathbf{P}$ & K & $\mathbf{C a}$ & Mg & \multirow{2}{*}{ Autor } \\
\hline & & & & $\left(\mathrm{kg} \mathrm{ha}^{-1}\right)$ & & & \\
\hline \multirow{2}{*}{0,5} & M & $4,0(11,5)$ & $0,3(16,4)$ & $2,7(27,3)$ & $0,9(10,8)$ & $0,5(20,8)$ & \multirow{2}{*}{1} \\
\hline & $\mathrm{C}$ & $2,3(6,7)$ & $0,1(6,7)$ & $0,9(8,7)$ & $0,9(11,7)$ & $0,3(10,0)$ & \\
\hline \multirow{2}{*}{2,4} & $\mathrm{M}$ & $32,7(11,3)$ & $2,0(13,9)$ & $41,5(21,7)$ & $10,5(10,4)$ & $3,5(12,1)$ & \multirow{2}{*}{2} \\
\hline & C & $43,3(15,0)$ & $1,7(11,9)$ & $24,4(12,7)$ & $28,9(28,6)$ & $5,4(18,6)$ & \\
\hline \multirow{2}{*}{2,4} & $\mathrm{M}^{*}$ & $39,5(11,8)$ & $4,3(18,0)$ & $59,8(23,0)$ & $14,2(13,2)$ & $4,9(13,9)$ & \multirow{2}{*}{3} \\
\hline & $\mathrm{C}^{* *}$ & $50,0(14,9)$ & $2,1(8,8)$ & $33,7(13,0)$ & $34,7(32,2)$ & $7,0(19,9)$ & \\
\hline \multirow{2}{*}{2,4} & $\mathrm{M}$ & $20,3(15,1)$ & $1,2(14,9)$ & $28,6(27,4)$ & $6,3(9,4)$ & $2,1(13,1)$ & \multirow{2}{*}{4} \\
\hline & $\mathrm{C}$ & $27,5(11,1)$ & $1,0(12,5)$ & $11,2(10,7)$ & $15,1(22,6)$ & $2,8(17,1)$ & \\
\hline \multirow{2}{*}{4,0} & $\mathrm{M}$ & $73,7(21,0)$ & $4,2(27,0)$ & $60,6(30,0)$ & $133,8(50,0)$ & $21,0(37,0)$ & \multirow{2}{*}{4} \\
\hline & C & $75,3(22,0)$ & $2,6(27,0)$ & $42,2(21,0)$ & $55,2(21,0)$ & $12,9(22,0)$ & \\
\hline \multirow{2}{*}{6,0} & $\mathrm{M}$ & 95,2 & 10,4 & 44,3 & 57,0 & 16,1 & \multirow{2}{*}{5} \\
\hline & $\mathrm{C}$ & 102,2 & 3,3 & 29,5 & 138,0 & 12,4 & \\
\hline \multirow{2}{*}{8,0} & $\mathrm{M}$ & $153,7(29,8)$ & $5,4(31,5)$ & $107,2(67,4)$ & $110,7(28,6)$ & $37,1(45,6)$ & \multirow[b]{2}{*}{6} \\
\hline & $\mathrm{C}$ & $105,7(20,5)$ & $2,4(14,0)$ & $64,5(10,5)$ & $115,7(29,9)$ & $12,9(15,8)$ & \\
\hline
\end{tabular}

1 - Viera et al. (2013); 2 - Caldeira et al. (2001); 3 - Caldeira et al. (1999); 4 - Caldeira et al. (2000); 4 - Caldeira et al. (2014); 5 - Calil (2003); 6 - Barichello (2003); * madeira; ** casca.

Com base nos dados obtidos de diferentes estudos calculou-se a eficiencia de utilização biológica (EUB). Esse índice é obtido pela razão da biomassa seca pela quantidade de nutrientes acumulados na mesma.

Nas Figuras 1 e 2 observamos a EUB para o componente madeira e casca 
respectivamente. Nota-se que, à medida em que a idade aumenta, a EUB cresce proporcionalmente. Isso mostra por exemplo que aos 0,5 anos $1 \mathrm{~kg}$ de fósforo é capaz de produzir $2.000 \mathrm{~kg}$ de madeira, já em um povoamento de 8 anos, teremos $1 \mathrm{~kg}$ de fósforo a cada $15.000 \mathrm{~kg}$ (Figura 1). 0 mesmo raciocínio é válido para o componente casca (Figura 2).
Observa-se que a eficiência da planta aumenta conforme avança sua maturidade. Concluímos que em rotações mais longas, a exportação de nutrientes é minimizada se for levado em conta a quantidade em $(\mathrm{kg})$ de madeira ou casca produzida.

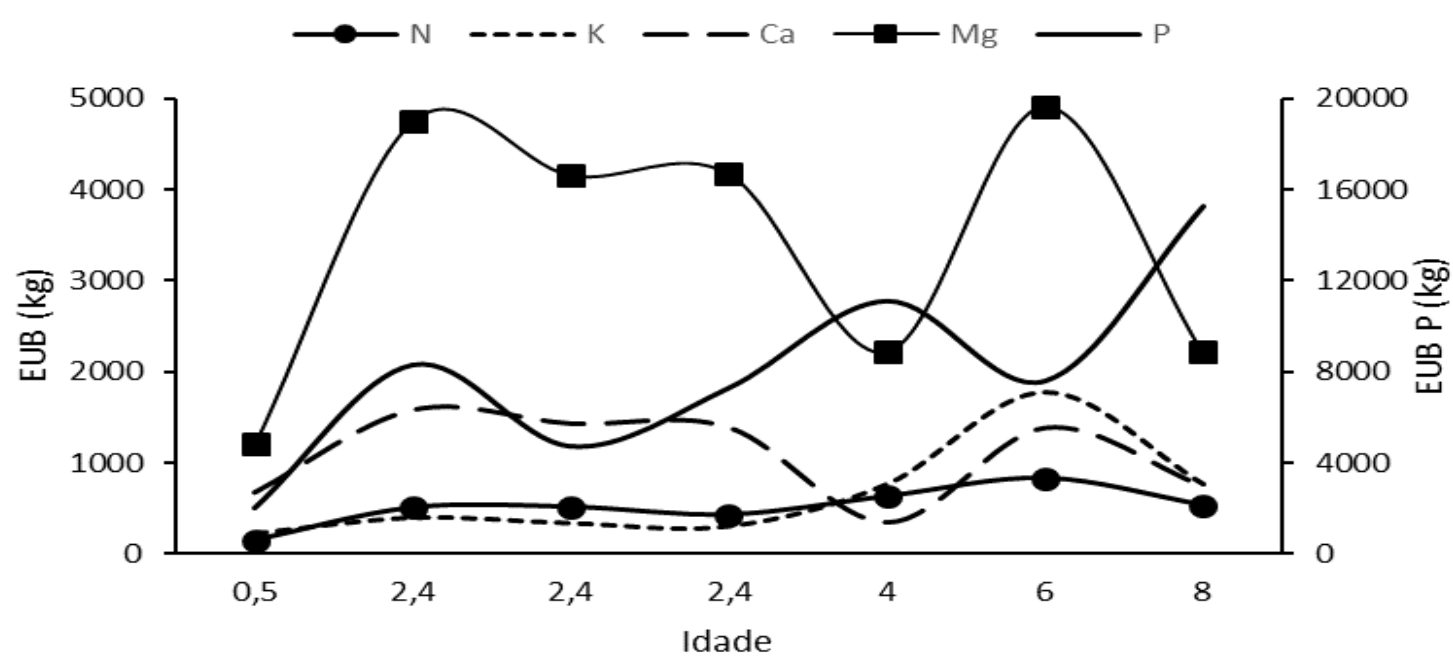

Figura 1. Eficiência de utilização biológica (EUN) da Acacia mearnsii para produção de madeira em diferentes idades.

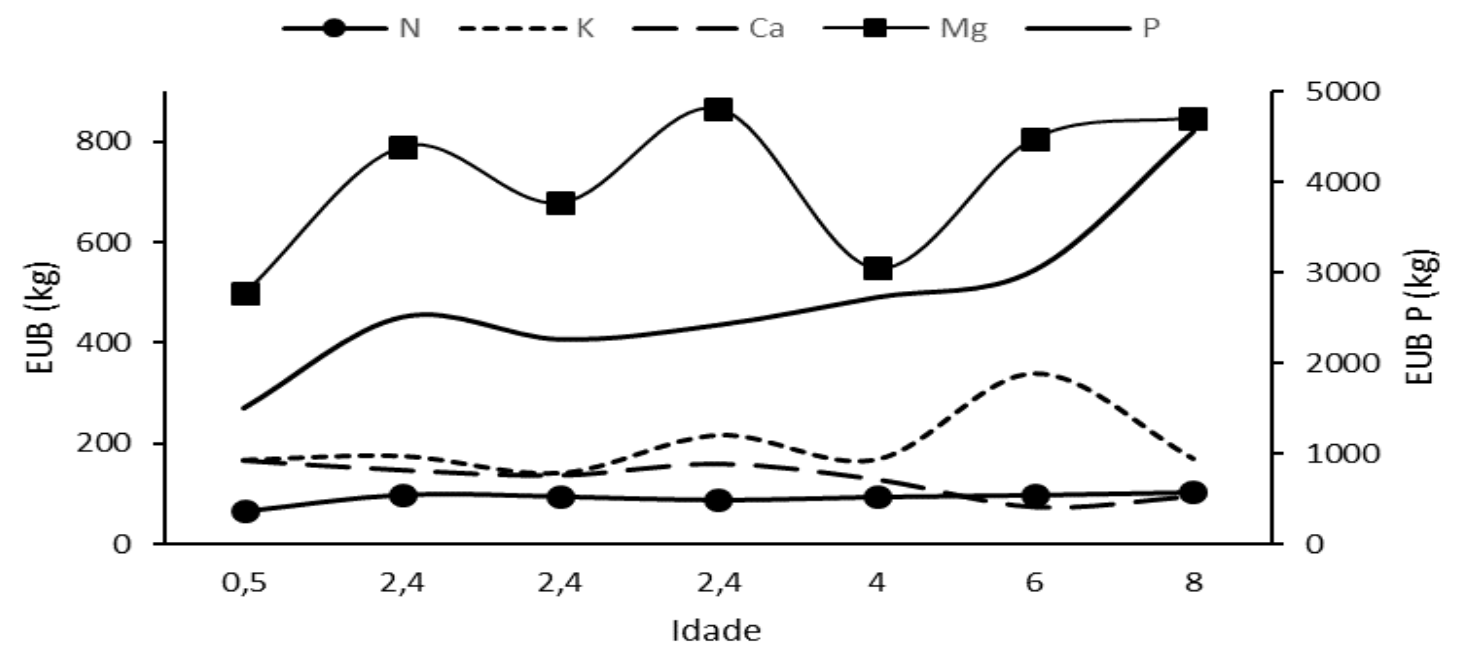

Figura 2. Eficiência de utilização biológica (EUN) da Acácia mearnsii para produção de casca em diferentes idades. 
0 fósforo é um dos nutrientes mais importantes na fertilização e o mais limitante nos solos brasileiros. Dessa forma a análise da EUB permite com que maximizemos a produção de madeira e casca levando em conta sua eficiência. A eficiência do $\mathrm{P}$ na madeira foi maior do que na casca. A explicação para tal se deve á alta mobilidade do elemento, sendo retranslocado dos tecidos mais velhos para os mais novos (Santana, 2005).

O comportamento inverso pode ser visto para o elemento cálcio (Ca). Sua baixa mobilidade nos tecidos da planta faz com que o mesmo acumule em tecidos mais velhos como o caso da madeira e da casca (König et al., 2002).

\section{Conflito de interesses}

Os autores declaram não haver conflito de interesses.

\section{Conclusões}

0 processamento do fuste a campo especialmente o descascamento de Acacia mearnsii permite a redução da exportação de nutrientes. A casca serve como fonte nutricional ás futuras rotações.

Sob ponto de vista sustentável, é desejável que a exportação de nutrientes seja mínima, bem como a quantidade de produto (madeira ou casca) seja maximizada. Para isso, a EUB serve como importante indicador, mostrando que em rotações com períodos maiores, obtémse maiores quantidades de produtos com a mesma quantidade de nutrientes.

\section{Referências}

AGEFLOR - Associação Gaúcha de Empresas Florestais. Anuário AGEFLOR 2016. Disponível em: <http://www.ageflor.com.br/ noticias/wpcontent/uploads/2016/09/AGEF LOR-DADOS-E-FATOS-2016.pdf>. Acesso em: 20 dez. 2018.

AGEFLOR - Associação Gaúcha de Empresas Florestais. A indústria de base florestal no
Rio Grande do Sul 2017. Porto Alegre: AGEFLOR, 2017.

Andrade, G. C.; Bellote, A. F. J.; Ferreira, C. A.; Silva, H. D. Adubação fosfatada e potássica em plantios de Acacia mearnsii de Wild. Boletim de Pesquisa Florestal, n. 48, p. 65-74, 2004.

Barichello, L. Quantificação da biomassa e dos nutrientes em floresta de Acacia mearnii De Wild. na Região Sul do Brasil. Santa Maria: Universidade Federal de Santa Maria, 2003. (Dissertação de Mestrado).

Barichello, L. R.; Schumacher, M. V.; Vogel, H. L. M. Quantificação da biomassa de um povoamento de Acacia mearnsii de Wild. na região sul do Brasil. Ciência Florestal, v. 15, n. 2, p. 129-135, 2005.

Boland, D. J.; Brooker, M. I. H.; Chippendale, G. M.; Hall, N.; Hyland, B. P. M.; Johnston, R. D.; Kleining, D. A.; Turner, J. D. Forest trees of Australia. Melbourne: Nelson-CSIRO, 1984.

Caldeira, M. V. W.; Rondon Neto, R. M.; Schumacher, M.V. Acúmulo e exportação de micronutrientes em um povoamento de acácia negra (Acacia mearnsii de Wild.) procedência Bodalla - Austrália. Revista Floresta, v. 33, n. 1, p. 73-78, 2003. https://doi.org/10.5380/rf.v33i1.2279

Caldeira, M. V.W.; Saidelles, F. L.F.; Schumacher, M. V.; Godinho, T. O. Biomassa de povoamento de Acacia mearnsii de Wild., Rio Grande do Sul, Brasil. Scientia Forestalis, v. 39, n. 90, p. 133-141, 2011.

Caldeira, M. V. W.; Schumacher, M. V.; Pereira, J. C.; Della-Flora, J. B.; Santos, E. M. Concentração e redistribuição de nutrientes nas folhas e no folhedo em um povoamento de Acacia mearnsii de Wild. no Rio Grande do Sul. Ciência Florestal, v. 9, n. 1, p. 19-24, 1999.

Caldeira, M. V. W.; Godinho, T. O.; Saidelles, F. L. F.; Viera, M.; Schumacher, M. V.; Castro, C. K. C. Exportação de carbono e nutrientes pela colheita de Acacia mearsii de Wild aos quatro anos de idade na Depressão Central, RS. Comunicata Scientiae, v. 5, n. 1, p. 68-74, 2014.

Caldeira, M. V. W.; Schumacher, M. V.; Santos, E. M.; Tedesco, N.; Pereira, J. C. Estimativa do conteúdo de nutrientes em um povoamento jovem de Acacia mearnsii De Wild. estabelecido na região sul do Brasil. Floresta, v. 29, n. 1/2, p. 53-65, 1999. 
Caldeira, M. V. W.; Schumacher, M. V.; Santos, E. M. Conteúdo de nutrientes em uma procedência de Acacia mearnsii plantada no Rio Grande do Sul-Brasil. Boletim de Pesquisa Florestal, n. 42, p. 105-120, 2001.

Caldeira, M. V. W.; Schumacher, M. V.; Tedesco, N.; Santos, E. M. Ciclagem de nutrientes em Acacia mearnsii De Wild. V. Ciclagem de nutrientes em Acacia mearnsii De Wild. V. Quantificação do conteúdo de nutrientes na biomassa aérea de Acacia mearnsii De Wild. procedência australiana. Ciência Rural, v. 30, n. 6, p. 977-982, 2000.

Calil, F. N. Aspectos da ciclagem de nutrientes em um sistema silvipastoril com Acacia mearnsii De Wild., no Município de Tupanciretã, RS. Santa Maria: Universidade Federal de Santa Maria, 2003. (Dissertação de Mestrado).

Caron, B. O.; Eloy, E.; Souza, V. Q.; Schmidt, D.; Balbinot, R.; Behling, A.; Monteiro, G. C. Quantificação da biomassa florestal em plantios de curta rotação com diferentes espaçamentos. Comunicata Scientiae, v. 6, n. 1, p. 106-112, 2015.

EMBRAPA - Empresa Brasileira de Pesquisa Agropecuária. Cultivo da acácia-negra. Sistemas de Produção, 2013. v. 3. Disponível em: <http://sistemasdeproducao.cnptia. embrapa.br/FontesHTML/AcaciaNegra/Culti vodaAcaciaNegra/>. Acesso em: 28 ago. 2017.

Forrester, D. I.; Bauhus, J.; Cowie, A. L. Nutrient cycling in a mixed-species plantation of Eucalyptus globulus and Acacia mearnsii. Canadian Journal of Forest Research, v. 35, n. 1, p. 2942-2950, 2005.

IBÁ - Indústria Brasileira de Árvores. Relatório anual 2017: ano base 2016. Brasília: IBÁ, 2017.

Inagaki, M.; Kamo, K.; Miyamoto, K.; Titin, J.; Jamalung, L.; Lapongan, J.; Miura, S. Nitrogen and phosphorus retranslocation and $\mathrm{N}: \mathrm{P}$ ratios of litterfall in three tropical plantations: Luxurius $\mathrm{N}$ and efficient $\mathrm{P}$ use by Acacia mangium. Plant and Soil, v. 341, p. 295-307, 2011. http://doi.org/10.1007/ s11104-010-0644-3

Kleinpaul, I. S.; Schumacher, M. V.; Viera, M.; Navroski, M. C. Plantio misto de Eucalyptus urograndis e Acacia mearnsii em sistema agroflorestal: produção de biomassa. Ciência
Florestal, v. 20, n. 4, p. 621-627, 2010. https://doi.org/10.5902/198050982420

König F. G.; Schumacher, M. V.; Brun, E. J.; Seling, I. Avaliação da sazonalidade da produção de serapilheira numa Floresta Estacional Decidual no Município de Santa Maria-RS. Revista Árvore, v. 26, n. 4, p. 429435, 2002.

Kull, C. A.; Shackleton, C. M.; Cunningham, P. J.; Ducatillon, C.; Dufour-Dror, J. M.; Esler, K.; Friday, J. B.; Gouveia, A. C.; Griffin, A. R.; Marchante, E.; Midgley, S. J.; Pauchard, A.; Rangan, H.; Richardson, D. M.; Rinaudo, T.; Tassin, J.; Urgenson, L. S.; von Maltitz, G. P.; Zenni, R. D.; Zylstra, M. J. Adoption, use, and perception of Australian acácias around the world. Diversity and Distributions, v. 17, n. 1, p. 822-836, 2011. https://doi.org/ 10.1111/j.1472-4642.2011.00783.x

Mochiutti, S.; Higa, A. R.; Simon, A. A. Fitossociologia dos estratos arbóreo e de regeneração natural em um povoamento de acácia-negra (Acacia mearnsii De Wild.) na região da floresta estacional semidecidual do Rio Grande do Sul. Ciência Florestal, v. 18, n. 1, p. 207-222, 2008.

Oliveira, H. A. Acácia-negra e tanino no Rio Grande do Sul. Canoas: La Salle, 1968.

Pallardy, S. Physiology of woody plants. San Diego: Academic Press, 2008.

Rachwal, M. F. G.; Curcio, G. R.; Dedecek, R. A. A influência das características pedológicas na produtividade de acácia-negra (Acacia mearnsii), Butiá, RS. Pesquisa Florestal Brasileira, n. 56, p. 53-62, 2008.

Richards, A. E.; Forrester, D. I.; Bauhus, J.; Scherer-Lorenzen, M. The influence of mixed tree plantations on the nutrition of individual species: A review. Tree Physiology, v. 30, n. 1, p. 1192-1208, 2010. https://doi.org/ 10.1093/treephys/tpq035

Richardson, D. M.; Rejmánek, M. Trees and shrubs as invasive alien species: A global review. Diversity and Distributions, v. 17, n. 1 , p. 788-809, 2011. https://doi.org/ 10.1111/j.1472-4642.2011.00782.x

Sanquetta, C. R.; Behling, A.; Corte, A. P. D.; Simon, A.; Pscheidt, H.; Ruza, M. S.; Mochiutti, S. Estoque de biomassa e carbono em povoamentos de acácia negra em diferentes idades no Rio Grande do Sul. Scientia Forestalis, v. 42, n. 103, p. 361-370, 2014. 
Santana, J. A. S. Estrutura fitossociológica, produção de serapilheira e ciclagem de nutrientes em uma área de Caatinga no Seridó do Rio Grande do Norte. Areia: Universidade Federal da Paraíba, 2005. (Tese de doutorado).

Schumacher, M. V.; Witschoreck, R.; Calil, F. N. Biomassa em povoamentos de Eucalyptus spp. de pequenas propriedades rurais em Vera Cruz, RS. Ciência Florestal, v. 21, n. 1, p.17-22, 2011. https://doi.org/10.5902/ 198050982743

Schumacher, M. V.; Viera, M.; Londero, E. K.; Calil, F. N.; Lopes, V. G.; Witschoreck, R. Crescimento da acácia-negra em resposta a aplicação de nitrogênio, fósforo e potássio. Cerne, v. 19, $\quad$ n. 1, p. 51-58, 2013. https://doi.org/10.1590/S0104-776020130 00100007

Silva, C. F.; Carmo, E. R.; Martins, M. A.; Freitas, M. S. M.; Pereira, M. G.; Silva, E. M. R. Deposition and nutritional quality of the litter of pure stands of Eucalyptus camaldulensis and Acacia mangium. Bioscience Journal, v. 31, n. 4, p. 1081-1091, 2015. https://doi.org/10.14393/BJ-v31n4 a2015-26297

Silva, D. A. A.; Silva, F. S. B.; Yano-Melo, A. M.; Maia, L. C. Uso de vermicomposto favorece o crescimento de mudas de gravioleira (Annona muricata L. 'Morada') associadas a fungos micorrízicos arbusculares. Acta Botânica Brasilica, v. 22, n. 3, p. 863-869, 2008. https://doi.org/10.1590/S0102-3306 2008000300021
Tedesco, N. Produção de mudas de acácianegra (Acacia mearnsii De Wild.) adubadas com N-P-K. Santa Maria: Universidade Federal de Santa Maria, 1999. (Dissertação de mestrado).

Torres, J. L. R.; Pereira, M. G. Dinâmica do potássio nos resíduos vegetais de plantas de cobertura no cerrado. Revista Brasileira de Ciência do Solo, v. 32, p. 1609-1618, 2008. https://doi.org/10.1590/S0100-06832008 000400025

Vargas, L. K. Lisboa, B. B.; Scholles, D.; Silveira, J. R. P.; Jung, G. C.; Granada, C. E.; Neves, A. G.; Braga, M. M.; Negreiros, T. Diversidade genética e eficiência simbiótica de rizóbios noduladores de acácia-negra de solos do Rio Grande do Sul. Revista Brasileira de Ciência do Solo, v. 31, p. 647654, 2007. https://doi.org/10.1590/S010006832007000400005

Viera, M.; Schumacher, M. V. Deposição de serapilheira e de macronutrientes em um povoamento de acácia-negra (Acacia mearnsii De Wild.) no Rio Grande do Sul. Ciência Florestal, v. 20, n. 2, p. 225-233, 2010. https://doi.org/10.5902/198050981848

Viera, M.; Schumacher, M. V.; Caldeira, M. V. W.; Watzlawick, L. F. Teores de nutrientes em povoamentos monoespecíficos e mistos de Eucalyptus urograndis e Acacia mearnsii em sistema agrossilvicultural. Ciência Florestal, v. 23, n. 1, p. 67-76, 2013. https://doi.org/ $10.5902 / 198050988440$ 